\title{
Periapical Cemento-osseous Dysplasia Is Rarely Diagnosed on Orthopantomograms of Patients with Neurofibromatosis Type 1 and Is Not a Gender-specific Feature of the Disease
}

\author{
REINHARD E. FRIEDRICH ${ }^{1,2}$ and ANIKA REUL ${ }^{1,2,3}$ \\ Departments of ${ }^{1}$ Oral and Craniomaxillofacial Surgery, and ${ }^{3}$ Prosthodontics, \\ Eppendorf University Hospital, University of Hamburg, Hamburg, Germany; \\ ${ }^{2}$ Neurofibromatosis Laboratory, Hamburg-Lokstedt, Hamburg, Germany
}

\begin{abstract}
Several skeletal aberrations of the skull have been described for the tumor predisposition syndrome neurofibromatosis type 1 (NF1). Recently, periapical cemental/cemento-osseous dysplasia (COD) has been described in females affected with NF1. This reactive lesion of the hard tissues in tooth-bearing areas of the jaw has been proposed to represent a gender-specific radiological feature of NF1. The aim of this study was to investigate the prevalence of $C O D$ in patients with NF1. Patients and Methods: The orthopantomograms (OPGs) of 179 patients with a confirmed diagnosis of NF1 were analyzed for $C O D$. The results were compared to radiographic findings obtained in OPGs of age-and sex-matched controls. The NF1 patient group was further differentiated according to the evidence of facial plexiform neurofibroma. Results: $C O D$ was a very rare finding in both groups. The extension of the diagnostic criteria including radiologically-healthy teeth and a widened periodontal gap in the periapical area only marginally increased the number of considered cases. Although there was a somewhat more common occurrence of such changes in the patient group compared to the control group and the number of affected women was greater than the number of men, none of these differences reached statistical significance. Furthermore, COD or widening of the periradicular periodontal space was not found to be associated with facial tumor type in NF1. Conclusion: The investigation revealed that $C O D$ is not a diagnostic feature
\end{abstract}

Correspondence to: Reinhard E. Friedrich, Prof. Dr. med., FEBOMFS, Department of Oral and Craniomaxillofacial Surgery, Eppendorf University Hospital, University of Hamburg, Martinistr. 52, 20246 Hamburg, Germany. Tel: +49 40741053259, e-mail: rfriedrich@uke.de

Key Words: Neurofibromatosis type 1, cemento-osseous dysplasia, periapical cemental dysplasia, gender difference. of NF1. There is no clear association of the rare finding of COD with gender. These studies should be compared with patient groups of other ethnic backgrounds.

Neurofibromatosis type 1 (NF1) is a tumor-suppressor gene disorder (1) with a large number of neoplastic and hamartous disorders of the body (2). It has been recognized that this disease also affects the skeleton $(3,4)$. The cranium is particularly affected by sphenoid bone dysplasia (5) and ossification disorders of the skull sutures, in particular the lambdoid (6). There are concepts linking the skeletal changes in NF1 to the function of the defective gene, whether by direct effects of gene product neurofibromin on bone organization and reorganization, or by the interaction of NF1-associated tumors with the adjacent skeleton $(4,7)$. The face is particularly affected in patients with NF1 when a facial plexiform neurofibroma (PNF) has developed (8). In these cases, an adjacent skeletal deformation of the jaws and other bones of the facial skull are regularly detected $(9,10)$. The extent of this deformation usually corresponds to the approximate course of the PNF-affected branch of the trigeminal nerve (11). There are almost always one-sided findings (10). The retention of teeth and numerical aberrations of permanent dentition are also reported in this disease (8). Recently, a Finnish group discovered another osseous finding related to NF1: periapical cemental dysplasia, also known as periapical cemento-osseous dysplasia (COD), a harmless and probably reactive lesion of the tooth-bearing jaw regions, was shown to be unusually common in female patients with NF1 (12). COD belongs to the group of fibro-osseous lesions of the jaws and is classified as an entity of (cemento)-osseous lesions $(13,14)$. From the evidence of many female patients with NF1 with COD in that study, it was concluded that COD was the first gender-specific feature of this autosomal dominant disease (12) (for this entity, the authors had used the term periapical cemental dysplasia). However, the finding of the increased 
occurrence of COD was not confirmed in a follow-up study by the same research group (15), as highlighted in a review of the radiographic characteristics of COD and other fibroosseous lesions of the jaw (16). Nonetheless, the increased occurrence of COD in female patients with NF1 remains the only widely recognized gender-specific diagnostic feature of the disease (17). Due to our many years of experience in the treatment of patients with NF1, we were unable to confirm this accumulation of lesions in patients with NF1 on the basis of case-by-case radiological findings. The aim of this study was therefore to review the prevalence of COD in NF1 using a large group of patients.

For the analysis of our own findings, it was essential to work critically on the repeatedly modified terminology of fibro-osseous lesions of the jaw published by committees of the World Health Organization (WHO) (18-20) and apply an easily verifiable classification of X-ray findings for the stated purpose.

\section{Materials and Methods}

Patients. Total cohort: In this study, 358 orthopantomograms (OPGs) obtained from 358 patients were evaluated (mean age $=34.63$ years; range $=12.57-69.13$ years).

Patient group: The OPGs of 179 patients of Caucasian origin with NF1 were investigated (NF1 patient group: mean age $=34.84$ years; range $=12.83-68.89$ years; $44.13 \%$ male $(n=79), 55.86 \%$ female $(n=100)$. These patients had been radiologically investigated for dental diseases and skeletal anomalies at the Department of Oral and Craniomaxillofacial Surgery, Eppendorf University Hospital, University of Hamburg, Germany. All patients fulfilled the updated diagnostic criteria for NF1 (2) and were citizens of Germany. Patients with a history of surgical skeletal procedures or trauma of the facial skeleton were excluded from evaluation. Many patients had carious or prosthetic or conservatively inadequately treated teeth. Since these patients originated from all federal states of the republic and the patients were referred for the treatment of nerve sheath tumors, the patients were usually informed of their dental findings and referred for dental care of their home region.

NF1 diagnostic groups. From this collection of OPGs of patients with NF1, subgroups were defined according to the tumor typology of the facial nerve sheath tumors: a total of 112 patients $(62.60 \%)$ showed disseminated cutaneous neurofibroma (DNF) but no facial $\mathrm{PNF}$, as revealed by physical inspection and complementary imaging [computed tomography (CT), magnetic resonance imaging (MRI) and B-scan ultrasound], if available, and also histology in the course of surgical treatment (REF) for neurogenic tumors (DNF group). OPGs of a further 67 patients $(37.43 \%)$ who showed histologically verified facial PNF were evaluated (facial PNF group, right side: 33 patients, left side: 34 patients). A patient was considered as having a facial PNF and categorized in this group if any facial region was affected by this type of tumor, irrespective of whether further DNF occurred in this region. The extent of visible infiltration of the PNF into the cutaneous territory of the nerve varied considerably. The PNFs were assigned to the facial cutaneous territories of the trigeminal nerve's three branches by analyzing facial photographs for tumor extension and consulting surgical reports, including remarks on the findings of tumor extension (REF). Subgroups of patients with facial PNF were identified according to the nerve branch or branches affected by this tumor. Visible cutaneous spread was the leading finding for this assignment. All facial PNFs showed a unilaterally restricted growth pattern (21).

Reference group. The reference group consisted of 179 OPGs of age- and sex-matched individuals that were selected from the archives of the Institute of Diagnostic Radiology in Dentistry of Hamburg University Dental Clinic. The age of these individuals was 12.57-69.13 years (mean=34 years). Patients with a history of facial trauma, genetic or bone disease or a history of jaw surgery were excluded from evaluation.

Dental treatment in both groups. The dental status of the patients was assumed to be given, without further conclusions being drawn from the X-ray image on pathologies that could have influenced the radiological presentation of the periodontal space [e.g. bruxism, overload of (frontal) teeth due to missing prosthetic support zones in the area of the molars and premolars]. These clinical conditions for the respective X-ray exposure were also applied to the cases of the reference group, since only the listed exclusion criteria were used for the selection of X-ray images of this group.

Ethics. All patients gave informed consent to the scientific study of their X-ray images and the evaluation of medical findings. This study was approved by the local University authority as a prerequisite to fulfilling the requirement of a dissertation in dentistry (AR). All procedures performed in this study involving human participants were in accordance with the ethical standards of the institutional and national research committee and with the 1964 Declaration of Helsinki and its later amendments or comparable ethical standards. Data were anonymized prior to analysis, and the investigators studying the radiographs were blinded to the diagnosis, the identity of individuals, and the assignment of the single case to a diagnostic group. The investigations of anonymized data were performed in accordance with Hamburgisches Gesundheitsdienstgesetz (Hamburg Health Service Act).

Equipment. Several panoramic X-ray devices were used in this Department during the recruitment period (1992-2010). Furthermore, OPGs from other institutions which had presented outpatients were also evaluated. However, the technical principles and the imaging characteristics were identical for all OPGs. The orientation of the skull on the radiological device in the spatial dimensions was ensured by adjustable light sources (8-10). However, this X-ray imaging technique is sensitive to deviations of the object from the focal trough (22). Imaging quality limitations should be taken into account for the assessment of the periradicular regions on OPGs.

$C O D$. In this study, COD is understood to mean a lesion focused on the alveolar process of the jaw (23) which has caused distinct but characteristic radiological alterations of the jaws both in the area of the tooth roots and in the area of previously tooth-bearing jaw sections. The term periapical cemental dysplasia (12) is synonymous with 'focal' COD or 'periapical' COD in this study. In principle, COD can also occur in edentulous areas of the jaws. COD was determined according to the radiological criteria as has been described in the literature (24-33). 
In this study, COD was assumed on OPG if the following criteria were met in a single tooth or several teeth: i. Free of caries and had not been treated prosthetically or conservatively with fillings; in other words, all teeth affected by caries or treated according to radiological criteria were excluded from the evaluation (34). ii. Lesion had a topographical relation to the root tip of a tooth $(32,33)$. iii. Lesion met the radiographic criteria of radiopacity or radiotransparency attributable to the three recognized stages of COD (27).

Since only a very few cases showed findings that met the criteria for COD in both study groups, teeth were included for which an apical enlargement of the periodontal space was conspicuous and thus could correspond to stage 1 of COD. As a result of this decision, teeth that had apical osteolysis in the intact tooth were considered, without this osteolysis developing a round configuration caudal to the apex and the center of the lesion outside of the apex (27). This extension of the diagnostic criteria meant that crescentshaped radiotranslucencies of the periradicular region of radiologically intact teeth were also evaluated when it could be clearly deduced from the radiograph that the diameter of the apical osteolysis narrowed towards the crown of the tooth to a normal periodontal space. These changes are referred to using the provisional term 'COD-like' structures.

In this study, radiopaque regions were present, both in the area of the alveolar processes and outside of these functional bone areas. These radiopaque lesions did not meet the criteria for COD as described above. A halo was not detectable at any of these radiopacities. These lesions were identified as either idiopathic osteosclerosis or condensing osteitis. These radiopaque findings were registered in each case and compared between the two groups.

In order to compare our own results with the data of the literature, several reviews and findings of individual reports were evaluated. The original description of the lesion of the respective publication is referred to.

Evaluation. The evaluation of the digital or digitized OPGs took place in a darkened room on a monitor approved for radiological assessment (35). The qualitative findings were limited to the judgment as to whether the specified radiographic findings were present in or absent from the individual case. The evaluation was carried out individually by each of the two investigators. Unclear cases were assessed together.

The radiological examination was carried out by the Authors who have more than 10 and 30 years of experience, respectively, in the evaluation of radiographs of the oral and maxillofacial regions. The correlative clinical findings on the radiological evaluations of all patients presented here have been raised by the senior Author himself.

Statistics. Chi-squared test and Fisher's exact test were used to calculate the differences between the values.

\section{Results}

The analysis was fraught with difficulty in identifying radiological findings in the study groups that looked at least similar to those of COD.

Several cases of peri-radicular widening of the periodontal space were demonstrated, possibly compatible with the first stage of COD (Figure 1). According to our own analysis, the apical findings of three patients of the NF1 group (ID 9, 97 and 102; Table I) and one case of the reference group (ID 252) were similar to COD stage 2 , for which a mixed radiopaque-radiotranslucent pattern is required in the alveolar process or edentulous areas (Figure 1).

In the patient group, there was no OPG in individuals with $\mathrm{X}$-ray findings that would have justified the diagnosis of stage 3 COD. Stage 3 COD cases would not have been registered in the evaluation even if root canal-treated teeth were included in this patient group. None of these cases of apical sclerosis in root canal-treated teeth with apical radiopacities (identified as reactive or condensing osteitis) had developed any radiotranslucent region around the sclerosis zone, thus lacking an essential feature of the radiological differential diagnosis of stage 3 COD. In the control group, there was one case of a homogeneous periradicular, oval zone of sclerosis of the mandibular left lateral incisor. This patient (ID 270) had pronounced bone loss due to periodontal disease. However, the incisor's radiopacity did not have the halo of a stage 3 COD and a hypercementosislike radiopacity was assessed (Figure 1). However, the cervical spine overlays occasionally make it difficult to assess changes in radiopacity in the anterior teeth.

The findings were summarized as COD-like structures. In total, there were seven cases in the NF1 patient group and four in the control group, which gives a prevalence of $3.91 \%$ (PNF group, $\mathrm{n}=1$; and DNF group, $\mathrm{n}=6$ ).

The findings were mainly collected for single teeth and almost all were restricted to the anterior mandible. In some cases, there were several affected teeth. None of the cases of apical crescent-shaped osteolysis of multiplg affected teeth involved more than two sextants; therefore, in our estimation, no case of the entire study group met the criteria for florid COD with respect to the extent of lesions. However, there are those who advocate bilateral occurrence as an inclusion criterion for florid COD (33).

No findings were seen in toothless areas that met the criteria of COD. In no case were radiopaque lesions found in toothless areas, which, due to their localization (above the nerve canal, residual alveolar process), would have required the differential diagnosis of stage 3 COD $v s$. idiopathic osteosclerosis. Indeed, isolated radiographic features of the jaws were identified in some further cases which were classified as idiopathic osteosclerosis. The relationship between these sclerotic zones, also known as dense bone islands, and the periapical area was very variable. The majority of sclerotic zones were located close to tooth roots without contact with them. Hypercementosis was an exceptional finding $(n=1)$. The number of radiopaque findings did not differ between the two study groups: five in the NF1 group (DNF, $n=2$ and PNF, $n=3$ ) and four in the reference group.

The differences in periapical findings in both groups were not significant, neither for the group of cases considered as 


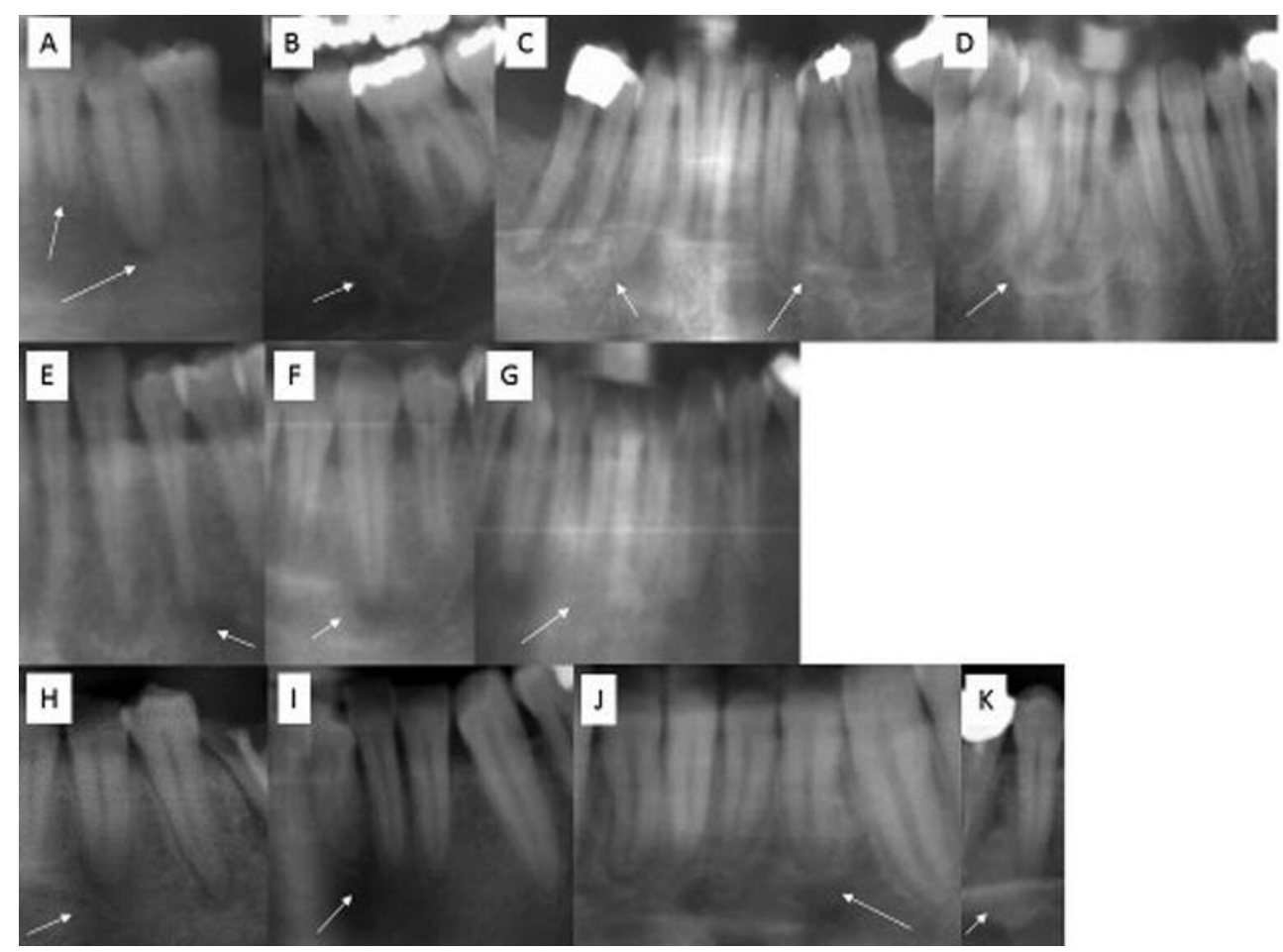

Figure 1. Cropped images of orthopantomograms illustrating findings consistent with the diagnosis of a cemento-osseous dysplasia (COD) or similar to those of the initial stages of COD. Arrows indicate the abnormal bone findings. Neurofibromatosis type 1 (NF1) group: A: Identity number (ID; see Table I) 8: tooth 31, 32 and 34; facial plexiform neurofibroma (PNF). B: ID 9: tooth 45 and 46; disseminated cutaneous neurofibroma (DNF). C: ID 97: frontal teeth, DNF. D: ID 102: frontal teeth, DNF. E: ID 129: tooth 32; 33, DNF. F: ID 175: tooth 33, DNF. G: ID 179: teeth 34-43; DNF. Reference group: H: ID 297: tooth 33 and 34. I: ID 250: teeth 32-42. J: ID 252: anterior teeth. K: ID 270: tooth 44. Numbering of teeth was according to the Fédération Dentaire Internationale.

stage 2 COD nor for the whole group of COD-like cases (Fisher's exact test $p>0.05$ for all variations and suspected entities).

\section{Discussion}

This study shows that COD was a rare finding in the study group in general and there did not appear to be any preference for the development of COD for patients with NF1 presented here. The more frequent occurrence of changes in periapical radiolucency in women is consistent with the well-known experience that such findings are more frequently registered in the general population for this sex $(16,32)$. In this study, COD of the jaw was neither an NF1associated phenomenon nor was there a statistically proven higher prevalence of periapical/periradicular COD-like X-ray findings in NF1-affected women compared to males.

The primary aim of this study was to determine whether findings similar to those of the Finnish working group would be observed (12). In the Finnish study, a significantly increased incidence of COD was detected, and COD was classified as the first gender-specific feature of NF1 (17). The association of COD with female sex in patients with NF1 was also subsequently critically considered by the authors themselves because they were unable to provide similar proof in another radiological study of the pathology of the facial skull in NF1-affected individuals (15).

The characteristic and convincing radiological findings of Visnapuu et al. (12) in the periapical area were not confirmed in this study. Stage 3 (focal=periapical) COD was not found in the NF1 group and the only debatable case of peri-radicular sclerosis in the reference group was very likely a hypercementosis in our opinion.

In order to avoid overlooking the initial stages of COD, further investigation also took into account periapical findings which only showed a widening of the periodontal space in this region, without the X-ray image necessarily indicating an approximately round periapical osteolysis. In other words, this extended registration of periapical findings also included those periradicular osteolyses whose imaginary center of idealized circular spread lay within the tooth root and therefore, only crescent-shaped osteolyses were imaged in the presence of 
Table I. Summary of radiological findings in individual patients and in the category of suspected cemento-osseous dysplasia of the alveolar process, in particular the peri-radicular regions. The prevailing radiological pattern was rated for classification to the category. The cases seen at stage 1 were predominantly characterized by some widening of the periapical space.

\begin{tabular}{|c|c|c|c|c|c|c|}
\hline Group & ID & Gender & $\begin{array}{l}\text { Age } \\
\text { (years) }\end{array}$ & $\begin{array}{c}\text { Stage } 1 \\
\text { radiotransparent }\end{array}$ & $\begin{array}{l}\text { Stage } 2 \text { radiopaque/ } \\
\text { radiotransparent }\end{array}$ & $\begin{array}{c}\text { Stage } 3 \\
\text { radiopaque }\end{array}$ \\
\hline \multirow[t]{7}{*}{ NF1 } & 8 & $\mathrm{M}$ & 33 & $X$ & & \\
\hline & 9 & $\mathrm{~F}$ & 30 & $X$ & & \\
\hline & 97 & $\mathrm{~F}$ & 43 & & $X$ & \\
\hline & 102 & F & 22 & & $X$ & \\
\hline & 129 & $\mathrm{~F}$ & 38 & & $\mathrm{X}$ & \\
\hline & 175 & M & 31 & $X$ & & \\
\hline & 179 & $\mathrm{~F}$ & 22 & $\mathrm{X}$ & & \\
\hline \multirow[t]{4}{*}{ Reference } & 297 & F & 36 & $\mathrm{X}$ & & \\
\hline & 250 & $\mathrm{~F}$ & 34 & $\mathrm{X}$ & & \\
\hline & 252 & M & 15 & $X$ & & \\
\hline & 270 & $\mathrm{~F}$ & 36 & & & $(\mathrm{X})$ \\
\hline
\end{tabular}

ID: Identity number; F: female; M: male.

intact apices. For this reason, all such defined lesions were included in the assessment; however, even with these numbers, there were no statistical differences in periapical findings between the groups. In addition, with regard to gender distribution, two out of the seven potential cases within the NF patient group were male, meaning that no genderspecific distribution of findings was demonstrated here either.

Limitations of this study. This investigation has important and clear limitations. The first is that the analysis was based solely on radiographic images. It was not possible to use clinical findings of the sensitivity of conspicuous teeth for these examinations. Thus, the key criterion of the clinical diagnosis of COD was not available for this study. This restriction also applies to the findings of the reference group. These restrictions were the main reason for the decision to consider only those teeth which were assessed in the OPG as untreated and intact (34). This reliance on restrictive inclusion criteria may lead to the inadvertent exclusion of endodontically treated teeth with persistent periradicular findings suggestive of COD (32).

The second limitation of the validity of the findings is the lack of histological confirmation of the radiological assessment. COD has a characteristic histology that is the basis for classifying this lesion as a reactive-inflammatory entity (24-31). Fundamental work on the definition of this lesion has been conducted by comparing radiological and histological findings (24-31). However, COD is a lesion that does not normally require surgical treatment $(32,33)$. It has been pointed out that earlier studies, which were characterized by the comparison of radiological and histological findings, would hardly be justified nowadays because the surgical procedures would constitute an unnecessary injury to the patients in a situation in which radiological diagnostics provide sufficient certainty in assessing the lesion's biological quality and surgical intervention would not be undertaken (33).

Adequacy of the applied X-ray technology. This investigation was based exclusively on OPGs. The dependency of the imaging quality of the dento-alveolar structures on the positioning of the facial skull in the focal trough of this $\mathrm{X}$ ray apparatus is well known (22). OPGs are blurred compared to individual dental images with special consideration of the image sharpness of the periodontal space, but it has the advantage of imaging more of the alveolar process than is possible with single image acquisitions. Previous studies have demonstrated the equivalence of OPG versus single-tooth image techniques for displaying the periapical region (36). With the introduction of cone beam CT, a highly detailed radiological cross-sectional imaging technique is available for the imaging of osseous structures in the maxillofacial area. It may be necessary to revise the assessment of periodontal space and adjacent bone by cone beam CT (37). However, it is unlikely that sophisticated radiological imaging techniques would have yielded different results in the research topic discussed here.

Terminology of fibro-osseous lesions of the jaws. Of all the diagnostic groups describing fibro-osseous lesions of the face and skull, the group of 'osseous dysplasias' has undergone the most changes in the names of individual entities (33). For the development history of (synonymous) terms and the differentiation of radiological concepts, the reader is referred to the detailed overview of MacDonald for the period up to 2005. This review includes the revised WHO classification 
of head and neck tumors from that year (33). The authors of the latest update of the WHO classification for neoplasms of the head and neck region, which includes the group of fibroosseous lesions, have decided to re-recognize the hard tissue of the tooth root as part of the reactive lesion. Therefore, the WHO reintroduced the term 'cemento-osseous dysplasia' instead of the term 'osseous dysplasia' which was first introduced for the previous revision of the WHO classification in 2005 (38). For this reason, the terminology for the COD of the jaws is very variable, depending on the recommended name at the time of publication. In the context of discussing our own results, the following terms used in the literature are used synonymously: periapical cement(al) dysplasia (12), focal COD $(28,29,32), \operatorname{COD}(13,14)$, and bony/osseous dysplasia (of the jaw) (13, 14, 39). Alternatively, the term cement(o)-osseous lesion is also used to address this group of entities (40).

It is generally believed that periapical COD and focal COD represent the most common fibro-osseous lesions of the jaws (31). On the basis of indistinguishable histological findings, it was successfully proposed that the assignment of the lesion to a specific entity on the basis of the skeletal (radiological) topography be abandoned and that these lesions be uniformly addressed as COD (31). Both types of COD are considered two different terms for the same non-neoplastic lesion $(31,41)$. One reason for maintaining both terms is that some authors diagnose periapical CODs when this alteration of the jaw has developed around the root apex of a vital tooth. In contrast, they prefer the term 'focal COD' to describe radiologically similar changes in the alveolar process or residual jaw, where the extraction of one or more teeth preceded. However, this differentiated terminology is not uniformly applied, therefore isolated periapical findings compatible with a diagnosis of periapical COD were addressed as focal COD (42). Indeed, focal COD has been defined as a solitary lesion (28). The terminological difficulties persist when other authors reserve the term 'periapical cemental dysplasia' exclusively for multilocular alterations of radiotransparent character restricted to the apices of vital mandibular anterior teeth (43). In fact, this term would apply to most of our own findings. In contrast, in a detailed Canadian study on osseous COD, this lesion was predominantly diagnosed in the mandibular posterior teeth (13, 14). These examples illustrate the authors' own reasons to refer to an easily verifiable terminology that allows for later falsification. The harmonization of the biology of these lesions with terminology is a contemporary task (44).

Age. Most individuals affected by COD are adults in the third to f'ifth decades of life $(32,33)$. Due to the inclusion criteria of the two study groups, adults were predominantly examined in this study. The age structure is similar to other studies on this topic. The cases with COD or COD-like radiographic findings matched the preferred age group of this lesion.
Gender. COD are predominantly detected in women (33). This finding also applies to our own examination. However, men also had peri-radicular lesions classified as COD or COD-like. Therefore, the findings do not allow our group to report any preferred appearance of these lesions in one sex.

Localization. COD is a lesion restricted to tooth-bearing parts of the jaws. These lesions are confined to the alveolar process and in the mandible they lie above the inferior dental canal (23). This description also applies to this investigation. Lesions of this type outside these bone areas were detected. The significant jaw deformities associated with PNF have been described elsewhere (8). The different terminology, derived from the topography and the tooth status, was simplified to a term according to uniform definitions (see above). However, COD may vary from non-expansive and asymptomatic to being expansive and symptomatic, the latter combination being quite rarely reported (45). These cases are apparently rare and cause diagnostic problems (46). The phenotype for expansive COD may vary considerably, can cause problems in identifying the correct diagnosis and may require complex surgical procedures (47). Such extensive lesions did not occur in this study.

Epidemiology. COD is a jaw lesion that is particularly prevalent in African populations and predominantly affects women (32). In populations of African descent, florid forms of COD are particularly common, regardless of the geography of the population (48). The two presented study groups were of Caucasian descent and thus do not belong to an ethnic group in which COD accumulates.

The prevalence of COD ranges between $0.4 \%$ and $3.2 \%$ (49-51). However, the evaluation of these data should take into account that the definition(s) of fibro-osseous lesions have changed over time. MacDonald points out that the reported cases of COD are unlikely to indicate the true incidence of this lesion $(32,33)$. If only the mixed radiolucent/opaque lesions are evaluated, the prevalence is $0.55 \%$ in the reference group and $1.67 \%$ in the group with NF1 in the presented study, and thus within the range of published values.

Pathogenesis. COD is a disease with an unknown origin. There are those who assume an exclusively osseous origin for these lesions (40) and those who speak of an odontogenic cause (23). This study does not help to clarify the cause of the disease, but it is very unlikely that the genetic elements that are essential to the tumor-suppressor gene disorder NF1 play a role in the development of COD.

Association of COD with other entities affecting the jaws. The occurrence of COD with other osseous and odontogenic lesions has been described, e.g. simple bone cysts (13) or odontogenic tumors/hamartomas (reviewed in 32). Such lesions were not detected in this study. 
Differential diagnosis. The most important differential diagnoses of COD are ossifying (cemento-ossifying) fibroma and osteomyelitis of the jaw (31). In none of the cases of either group listed here were these diagnoses considered (see exclusion criteria). The distinction of the initial stages of COD from an apical inflammatory response is probably one of the most common clinical tasks in the analysis of clinical and radiological findings $(27,52)$.

Radiopacities. Radiopacities in this study were demonstrated in the tooth-bearing area as well as in the base of the mandible. However, the "target-like concentric arrangement of radiolucent and radiopaque areas" (53), (defining the sclerotic part of the mixed radiopaque/translucent stage of COD) was a rare phenomenon in both groups. In isolated osteosclerotic lesions, there was either no relation to the periodontium or the radiopacity was a reactive lesion on diseased teeth. This type of solitary osteosclerosis was not part of the questionable entity of this study. However, the preservation of the periodontal space on the radiograph as a criterion of initial COD has not yet been clarified $(27,34$, 53-55).

\section{Conclusion}

This study shows that COD is rare in patients suffering from the tumor-suppressor gene disorder NF1 and, in the few cases of this group who had radiological signs comparable to or in accordance with these lesions, there was no statistically significant gender predilection. COD of the jaws is not a radiological hallmark of NF1.

\section{References}

1 Rad E and Tee AR: Neurofibromatosis type 1: Fundamental insights into cell signalling and cancer. Semin Cell Dev Biol 52: 39-46, 2016

2 Ferner RE and Gutmann DH: Neurofibromatosis type 1 (NF1): Diagnosis and management. Handb Clin Neurol 115: 939-955, 2013.

3 Adrian C: Ueber Neurofibromatose und ihre Komplikationen. Beitr Klin Chir 31: 1-98, 1901.

4 Alwan S, Tredwell SJ and Friedman JM: Is osseous dysplasia a primary feature of neurofibromatosis 1 (NF1)? Clin Genet 67: 378-390, 2005.

5 Jacquemin C, Bosley TM and Svedberg H: Orbit deformities in craniofacial neurofibromatosis type 1. AJNR Am J Neuroradiol 24: 1678-1682, 2003

6 Arrington DK, Danehy AR, Peleggi A, Proctor MR, Irons MB and Ullrich NJ: Calvarial defects and skeletal dysplasia in patients with neurofibromatosis Type 1. J Neurosurg Pediatr 11: 410-416, 2013.

7 Riccardi VM: Neurofibromatosis type 1 is a disorder of dysplasia: the importance of distinguishing features, consequences, and complications. Birth Defects Res A Clin Mol Teratol 88: 9-14, 2010.
8 Friedrich RE, Giese M, Schmelzle R, Mautner VF and Scheuer HA: Jaw malformations plus displacement and numerical aberrations of teeth in neurofibromatosis type 1: a descriptive analysis of 48 patients based on panoramic radiographs and oral findings. J Craniomaxillofac Surg 31: 1-9, 2003.

9 Friedrich RE, Rother J, Christ G, Lehmann M, Eulenburg CG, Giese $M$ and Scheuer HA: Analysis of orbital plain radiographs for orbital deformities in neurofibromatosis type 1 patients, with special reference to alterations of the orbital rim as indicators of adjacent plexiform neurofibroma. Anticancer Res 33: 1081-1090, 2013.

10 Friedrich RE, Lehmann JM, Rother J, Christ G, Zu Eulenburg C, Scheuer HT and Scheuer HA: A lateral cephalometry study of patients with neurofibromatosis type 1. J Craniomaxillofac Surg 45: 809-820, 2017.

11 Loutfy WG, Ryan DE, Toohill RJ and Meyer GA: Trigeminal nerve neurofibroma: case report. J Oral Maxillofac Surg 48: 650654, 1990.

12 Visnapuu V, Peltonen S, Ellilä T, Kerosuo E, Väänänen K, Happonen RP and Peltonen J: Periapical cemental dysplasia is common in women with NF1. Eur J Med Genet 50: 274-280, 2007.

13 Chadwick JW, Alsufyani NA and Lam EW: Clinical and radiographic features of solitary and cemento-osseous dysplasiaassociated simple bone cysts. Dentomaxillofac Radiol 40: 230235,2011

14 Alsufyani NA and Lam EW: Cemento-osseous dysplasia of the jaw bones: key radiographic features. Dentomaxillofac Radiol 40: 141-146, 2011.

15 Visnapuu V, Peltonen S, Tammisalo T, Peltonen J and Happonen RP: Radiographic findings in the jaws of patients with neurofibromatosis 1. J Oral Maxillofac Surg 70: 1351-1357, 2012.

16 MacDonald DS: Maxillofacial fibro-osseous lesions. Clin Radiol 70: 25-36, 2015.

17 Javed F, Ramalingam S, Ahmed HB, Gupta B, Sundar C, Qadri $\mathrm{T}$, Al-Hezaimi $\mathrm{K}$ and Romanos GE: Oral manifestations in patients with neurofibromatosis type-1: a comprehensive literature review. Crit Rev Oncol Hematol 91: 123-129, 2014.

18 Kramer, IRH, Pindborg JJ and Shear M (eds.): Histological typing of odontogenic tumours. In: World Health Organization. International Histological Classification of Tumours. Springer, Berlin - Heidelberg, 1992.

19 Barnes L, Eveson JW, Reichart P and Sidransky D (eds.): World Health Organization (WHO) Classification of Tumours. Head and Neck Tumours. Pathology \& Genetics. WHO Press, Albany, NY, IARC, Lyon, 2005.

20 El-Naggar AK, Chan JKC, Grandis JR, Takata T and Slootweg PJ (eds.): WHO Classification of Head and Neck Tumours. Fourth edition. IARC WHO Classification of Tumours, No 9., IARC, Lyon, 2017.

21 Friedrich RE, Giese M, Stelljes C, Froeder C and Scheuer HA: Size of tooth crowns and position of teeth concerning the extension of facial plexiform neurofibroma in patients with neurofibromatosis type 1. Anticancer Res 32: 2207-2214, 2012.

22 Riecke B, Friedrich RE, Schulze D, Loos C, Blessmann M, Heiland $\mathrm{M}$ and Wikner $\mathrm{J}$ : Impact of malpositioning on panoramic radiography in implant dentistry. Clin Oral Investig 19: 781-790, 2015

23 Melrose RJ: The clinico-pathologic spectrum of cemento-osseous dysplasia. Oral Maxillofac Clin North Am 9: 643-653, 1997. 
24 Waldron CA and Giansanti JS: Benign fibro-osseous lesions of the jaws: a clinical-radiologic-histologic review of sixty-five cases. Oral Surg Oral Med Oral Pathol 35: 190-201, 1973.

25 Waldron CA and Giansanti JS: Benign fibro-osseous lesions of the jaws: a clinical-radiologic-histologic review of sixty-five cases. II. Benign fibro-osseous lesions of periodontal ligament origin. Oral Surg Oral Med Oral Pathol 35: 340-350, 1973.

26 Waldron CA, Giansanti JS and Browand BC: Sclerotic cemental masses of the jaws (so-called chronic sclerosing osteomyelitis, sclerosing osteitis, multiple enostosis, and gigantiform cementoma. Oral Surg Oral Med Oral Pathol 39: 590-604, 1975.

27 Shafer WG, Hine MK and Levy BM: A Textbook of Oral Pathology, Fourth Edition, Saunders, Philadelphia, pp. 297-299, 1983.

28 Summerlin DJ and Tomich CE. Focal cemento-osseous dysplasia: a clinicopathologic study of 221 cases. Oral Surg Oral Med Oral Pathol 78: 611-620, 1994.

$29 \mathrm{Su}$ L, Weathers DR and Waldron CA: Distinguishing features of focal cemento-osseous dysplasias and cemento-ossifying fibromas: I. A pathologic spectrum of 316 cases. Oral Surg Oral Med Oral Pathol Oral Radiol Endod 84: 301-309, 1997.

$30 \mathrm{Su}$ L, Weathers DR and Waldron CA: Distinguishing features of focal cemento-osseous dysplasia and cemento-ossifying fibromas. II. A clinical and radiologic spectrum of 316 cases. Oral Surg Oral Med Oral Pathol Oral Radiol Endod 84: 540-549, 1997.

31 Eversole R, Su L and ElMofty S: Benign fibro-osseous lesions of the craniofacial complex. Head Neck Pathol 2: 177-202, 2008.

32 Macdonald-Jankowski DS. Focal cemento-osseous dysplasia: a systematic review. Dentomaxillofac Radiol 37: 350-360, 2008.

33 MacDonald D: Oral and Maxillofacial Radiology, Wiley Blackwell, Chichester, 2011, p. 166, pp. 176-181.

34 Cavalcanti PHP, Nascimento EHL, Pontual MLDA, Pontual ADA, Marcelos PGCL, Perez DEDC and Ramos-Perez FMM: Cemento-osseous dysplasias: imaging features based on cone beam computed tomography scans. Braz Dent J 29: 99-104, 2018.

35 Friedrich RE, Bruhn $\mathrm{M}$ and Lohse C: Cone-beam computed tomography of the orbit and optic canal volumes. J Craniomaxillofac Surg 44: 1342-1349, 2016.

36 Tammisalo T, Luostarinen T, Vähätalo K and Neva M: Detailed tomography of periapical and periodontal lesions. Diagnostic accuracy compared with periapical radiography. Dentomaxillofac Radiol 25: 89-96, 1996.

37 Pope O, Sathorn C and Parashos P: A comparative investigation of cone-beam computed tomography and periapical radiography in the diagnosis of a healthy periapex. J Endod 40: 360-365, 2014.

38 Speight PM and Takata T: New tumour entities in the 4th edition of the World Health Organization Classification of head and neck tumours: Odontogenic and Maxillofacial Bone Tumours. Virchows Arch, 2017. doi: 10.1007/s00428-017-2182-3. [Epub ahead of print]

39 Mainville GN, Turgeon DP and Kauzman A: Diagnosis and management of benign fibro-osseous lesions of the jaws: a current review for the dental clinician. Oral Dis 23: 440-450, 2017.
40 Speight PM and Carlos R: Maxillofacial fibro-osseous lesions. Curr Diagn. Pathol 12: 1-10, 2006.

41 Hall G: Fibro-osseous lesions of the head and neck. Diagn Histopathol 18: 149-158, 2012.

42 Galgano C, Samson J, Küffer R and Lombardi T: Focal cementoosseous dysplasia involving a mandibular lateral incisor. Int Endod J 36: 907-911, 2003.

43 Schulz M, Reichart PA, Dula K and Bornstein MM: Focal osteosclerotic lesions of the jaws - A review focusing on diagnostic and therapeutic aspects. Schweiz Monatsschr Zahnmed 120: 325-332, 2010 (in German).

44 Noffke CE, Raubenheimer EJ and MacDonald D: Fibro-osseous disease: harmonizing terminology with biology. Oral Surg Oral Med Oral Pathol Oral Radiol 114: 388-392, 2012.

45 Malek M, Cortes LM, Sigurdsson A and Rosenberg PA: Differential diagnosis of a periapical radiolucent lesion. A case report and review of the literature. N Y State Dent J 81: 52-56, 2015.

46 Bulut EU, Acikgoz A, Ozan B, Zengin AZ and Gunhan O: Expansive focal cemento-osseous dysplasia. J Contemp Dent Pract 13: 115-118, 2012.

47 Raubenheimer EJ, Noffke CE and Boy SC: Osseous dysplasia with gross jaw expansion: A review of 18 lesions. Head Neck Pathol 10: 437-443, 2016

48 Owosho AA, Potluri A and Bilodeau EA: Osseous dysplasia (cemento-osseous dysplasia) of the jaw bones in western Pennsylvania patients: analysis of 35 cases. PA Dent J 80: 2529, 2013.

49 Matsuzaka K, Shimono M, Uchiyama T, Noma H and Inoue T: Lesions related to the formation of bone, cartilage or cementum arising in the oral area: a statistical study and review of the literature. Bull Tokyo Dent Coll 43: 173-180, 2002.

50 Stafne EC: Periapical osteofibrosis with formation of cementoma. J Am Dent Assoc 21: 1822-1829, 1934

51 Chaudhry AP, Spink JH and Gorlin RJ: Periapical fibrous dysplasis (cementoma). J Oral Surg 16: 483-488, 1958.

52 Roghi M, Scapparone C, Crippa R, Silvestrini-Biavati A and Angiero F: Periapical cemento-osseous dysplasia: clinicopathological features. Anticancer Res 34: 2533-2536, 2014.

53 Langland OE, Langlais RP and Morris CR (eds.): Principles and Practice of Panoramic Radiology. Saunders, Philadelphia, 1982, pp. 325-329.

54 Abramovitch K and Rice DD: Benign fibro-osseous lesions of the jaws. Dent Clin North Am 60: 167-193, 2016.

55 Ahmad M and Gaalaas L: Fibro-osseous and other lesions of bone in the jaws. Radiol Clin North Am 56: 91-104, 2018.

Received January 11, 2018

Revised February 9, 2018

Accepted February 14, 2018 